\title{
Convection mixte MHD d'un nanofluide (eau-Cu) dans une cavité ouverte
}

\author{
Fersadou Brahim ${ }^{1, *}$ Walid Nessab $^{1}$, et Henda Kahalerras $^{1}$ \\ ${ }^{1}$ B.P. 32, El Alia, Bab Ezzouar 16111, USTHB, Alger - Algérie
}

\begin{abstract}
Résumé. Dans la présente étude, le problème de la convection mixte MHD d'un nanofluide (eau - $\mathrm{Cu}$ ) confiné dans une cavité ouverte munie de deux sources de chaleur est étudié numériquement. Le modèle de Buongiorno est utilisé pour décrire l'écoulement du nanofluide en tenant compte du mouvement Brownien et de l'effet thermophorèse. Les équations gouvernantes avec les conditions aux limites associées sont résolues par la méthode des volumes finis. Les résultats révèlent un transfert de chaleur accru avec l'augmentation du rapport d'ouverture (R) et du nombre de Hartmann (Ha).

Mots-clés : Convection mixte, MHD, Nanofluide, Cavité ouverte
\end{abstract}

\section{Introduction}

La convection mixte est d'un intérêt crucial pour les chercheurs dans le domaine industriel tel que le refroidissement des composants électroniques de puissance. Ce mode de transfert thermique peut être amélioré passivement en employant diverses méthodes dont l'utilisation des nanofluides [1-3] ou encore en se servant des cavités ouvertes [4-6]. C'est dans ce contexte que s'inscrit ce travail qui consiste à examiner numériquement le problème de la convection mixte MHD d'un nanofluide (eau-Cu) dans une cavité ouverte en tenant compte des effets du mouvement Brownien et thermophorèse.

\section{Modèle physique et formulation mathématique}

Le domaine physique étudié, représenté sur la Figure 1 , est une cavité rectangulaire ouverte sur une largeur d constituée de parois électriquement isolées. Le rapport d'ouverture à l'entrée et à la sortie de la cavité est défini comme : $\mathrm{R}=\mathrm{H} / \mathrm{d}$. La plaque de gauche est refroidie avec un flux de chaleur constant $\mathrm{q}_{\mathrm{f}}$ tandis que celle de droite contient une alternance de zones adiabatiques et de sources de chaleur de flux thermique constante $\mathrm{q}_{\mathrm{c}}$. Un champ magnétique externe $\overrightarrow{\mathrm{B}}_{0}$ d'intensité uniforme est appliqué dans la direction transversale $\mathrm{y}$.

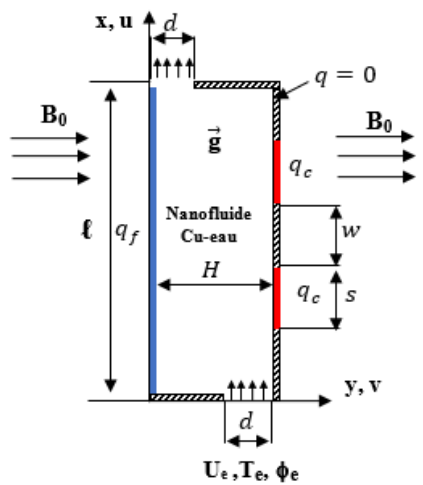

Fig. 1. Domaine physique

${ }^{1}$ Corresponding author: bfersadou@usthb.dz 
En adoptant le modèle de Buongiorno [7], les équations de conservation ainsi que les conditions aux limites peuvent être exprimées de la manière suivante :

$$
\begin{aligned}
& \frac{\partial U}{\partial X}+\frac{\partial V}{\partial Y}=0 \\
& U \frac{\partial U}{\partial X}+V \frac{\partial U}{\partial Y}=-\frac{\partial P}{\partial X}+\frac{1}{R \operatorname{Re}} \frac{\rho_{f}}{\rho_{n f} \mu_{f}}\left(\frac{\partial}{\partial X}\left(\mu_{n f} \frac{\partial U}{\partial X}\right)+\frac{\partial}{\partial Y}\left(\mu_{n f} \frac{\partial U}{\partial Y}\right)\right)+\frac{(\rho \beta)_{n f} \rho_{f}}{(\rho \beta)_{f} \rho_{n f}} R R i \theta-\frac{H a^{2}}{R e} \frac{\sigma_{n f} \rho_{f}}{\sigma_{f} \rho_{n f}} U \\
& U \frac{\partial V}{\partial X}+V \frac{\partial V}{\partial Y}=-\frac{\partial P}{\partial Y}+\frac{1}{R \operatorname{Re}} \frac{\rho_{f}}{\rho_{n f} \mu_{f}}\left(\frac{\partial}{\partial X}\left(\mu_{n f} \frac{\partial V}{\partial X}\right)+\frac{\partial}{\partial Y}\left(\mu_{n f} \frac{\partial V}{\partial Y}\right)\right) \\
& U \frac{\partial \theta}{\partial X}+V \frac{\partial \theta}{\partial Y}=\frac{1}{R \operatorname{Re} P r} \frac{\left(\rho C_{P}\right)_{f}}{\left(\rho C_{P}\right)_{n f}}\left(\frac{\partial}{\partial X}\left(k_{n f} \frac{\partial U}{\partial X}\right)+\frac{\partial}{\partial Y}\left(k_{n f} \frac{\partial U}{\partial Y}\right)\right)+\frac{E c H a^{2}}{R \operatorname{Re}} \frac{\left(\rho C_{P}\right)_{f}}{\left(\rho C_{P}\right)_{n f}} \frac{\sigma_{n f}}{\sigma_{f}} U^{2} \\
& +\frac{1}{R \operatorname{RePr}}\left[N_{B}\left(\frac{\partial \varphi}{\partial X} \frac{\partial \theta}{\partial X}+\frac{\partial \varphi}{\partial Y} \frac{\partial \theta}{\partial Y}\right)+N_{T}\left(\left(\frac{\partial \theta}{\partial Y}\right)^{2}+\left(\frac{\partial \theta}{\partial Y}\right)^{2}\right)\right] \\
& U \frac{\partial \varphi}{\partial X}+V \frac{\partial \varphi}{\partial Y}=\frac{1}{R \operatorname{RePr}}\left(\frac{\partial}{\partial X}\left(\frac{1}{L e} \frac{\partial \varphi}{\partial X}\right)+\frac{\partial}{\partial Y}\left(\frac{1}{L e} \frac{\partial \varphi}{\partial Y}\right)\right)+\frac{1}{R \operatorname{RePr}}\left(\frac{\partial}{\partial X}\left(\frac{N_{T}}{N_{B} L e} \frac{\partial \theta}{\partial X}\right)+\frac{\partial}{\partial Y}\left(\frac{N_{T}}{N_{B} L e} \frac{\partial \theta}{\partial Y}\right)\right)
\end{aligned}
$$

\section{Modélisation numérique}

Les équations gouvernantes sont résolues numériquement en utilisant la méthode des volumes finis [8]. Un maillage non uniforme est employé en plaçant les mailles les plus fines à l'entrée, à la sortie et au niveau des parois de la cavité. Le système de grille appliqué est de $250 \times 50$ (dans les directions $\mathrm{X}$ et $\mathrm{Y}$, respectivement).

\section{Résultats}

La Figure 2 montre les lignes de courants et les isothermes pour différents rapports d'ouverture. Pour $R \geq 2.5$, le jet se développe en suscitant du côté froid un bloc d'écoulement inverse animé par la force de flottabilité qui s'étend le long de la cavité en créant des zones de recirculation. La Figure 3 illustre l'évolution de $\mathrm{Nu}_{\mathrm{m} 1}, \mathrm{Nu}_{\mathrm{m} 2}$ et $\mathrm{Nu}_{\mathrm{g}}$ avec $\mathrm{R}$. Nous notons que le plus grand taux de refroidissement est obtenu pour $\mathrm{R}=10$ suite à l'écoulement rapide du nanofluide qui est alimenté d'une part, par le jet et d'autre part, par la force de flottabilité.

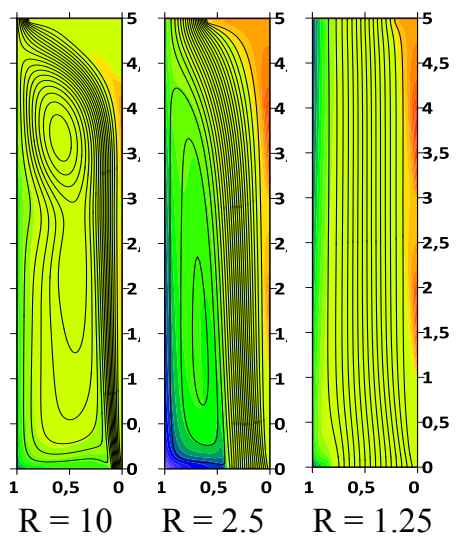

Fig. 2. Lignes de courant et isothermes pour différents rapports d'ouverture à $\mathrm{Ha}=0$ et $\mathrm{Ri}=2.5$

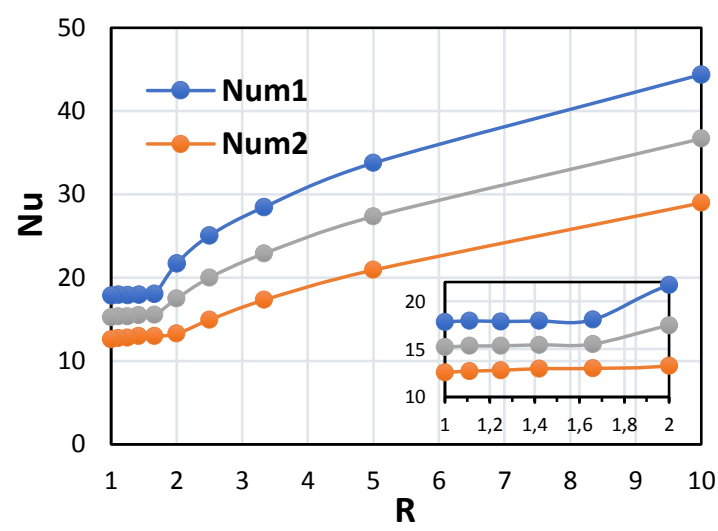

Fig. 3. Variation des nombres de Nusselt avec $\mathrm{R}$ à $\mathrm{Ha}=0$ et $\mathrm{Ri}=2.5$ 
Il apparait des lignes de courants de la Figure 4, que l'augmentation du nombre de Hartmann tend à ralentir le mouvement du nanofluide près de la paroi contenant les sources chaudes et à l'accélérer du côté de la paroi froide. Ce comportement est dû au fait que l'application du champ magnétique crée une résistance supplémentaire à l'écoulement due à la force de Lorentz agissant dans la direction verticale et dans le sens opposé au mouvement du nanofluide et à la force de flottabilité. Cette redistribution homogène de la structure d'écoulement améliore le transfert de chaleur, en particulier pour des forces de flottabilité élevées et à des $\mathrm{Ha}>10$ comme le montre la Fig.5.

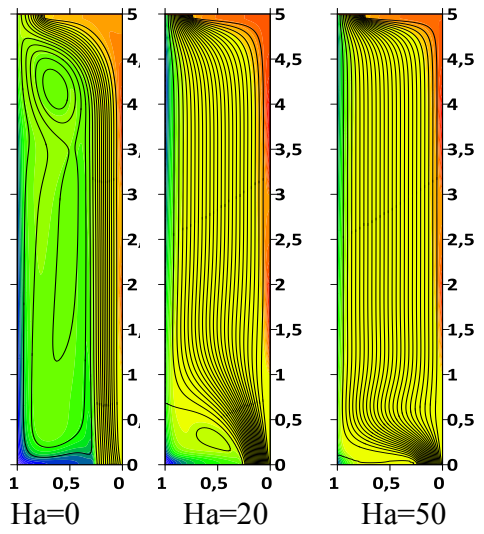

Fig. 4. Lignes de courant et isothermes pour différents $\mathrm{Ha}$ à $\mathrm{R}=4$ et $\mathrm{Ri}=2.5$

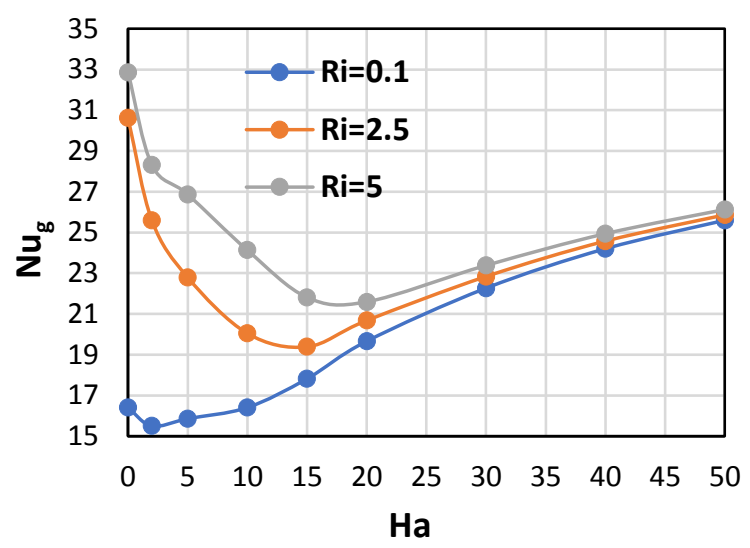

Fig. 5. Variation du nombre de Nusselt globale avec Ha pour différents nombres de $\mathrm{Ri}$ à $\mathrm{R}=4$

\section{Conclusion}

Le présent travail est une simulation numérique de la convection d'un nanofluide (eau $\mathrm{Cu}$ ) dans une cavité ouverte chauffée asymétriquement en présence d'un champ magnétique uniforme. L'analyse des effets du rapport d'ouverture (R), du nombre de Hartmann (Ha) et du nombre de Richardson (Ri), a permis de conclure que le transfert de chaleur est amélioré par la réduction de l'ouverture de la cavité. Les grandes valeurs du nombre de Hartmann assurent une homogénéisation de l'écoulement du nanofluide et un meilleur refroidissement des sources chaudes en particulier pour des Ri élevés.

\section{Références}

[1] M. Sheikholeslami, M. Hatami, D.D. Ganji. Powder Tech.246,327-336 (2013)

[2] A.A. Servati, K. Javaherdeh, H.R. Ashorynejad, Adv. Powder Tech.25, 666-675 (2014)

[3] I. Fersadou, H. kahalerras, M. El Ganaoui. Computers \& Fluids.121C, 164-179 (2015)

[4] Nirmalendu Biswas, Nirmal K. Manna. Int. J. Heat Mass Transfer. 114, 430-452 (2017)

[5] S. Hussain, S.E. Ahmed, T. Akbar. Int. J. Heat Mass Transfer. 114, 1054-1066 (2017)

[6] A.A.A.A. Al-Rashed, K. Kalidasan, L. Kolsi, R. Velkennedy, A. Aydi, A.K. Hussein,

E.H. Malekshah. Int. J. Mech. Sci.135, 362-375 (2018)

[7] J. Buongiorno. ASME J. Heat Transfer. 128, 240-250 (2006)

[8] S.V. Patankar. McGraw Hill (1980) 